Western University

Scholarship@Western

$1-15-2014$

\title{
Effect of age on the hemodynamic and sympathetic responses at the onset of isometric handgrip exercise.
}

Sophie Lalande

Carolyn P Sawicki

Jacquie R Baker

Kevin Shoemaker

The University of Western Ontario, kshoemak@uwo.ca

Follow this and additional works at: https://ir.lib.uwo.ca/kinpub

Part of the Kinesiology Commons

\section{Citation of this paper:}

Lalande, Sophie; Sawicki, Carolyn P; Baker, Jacquie R; and Shoemaker, Kevin, "Effect of age on the hemodynamic and sympathetic responses at the onset of isometric handgrip exercise." (2014).

Kinesiology Publications. 22.

https://ir.lib.uwo.ca/kinpub/22 


\title{
Effect of age on the hemodynamic and sympathetic responses at the onset of isometric handgrip exercise
}

\author{
Sophie Lalande, ${ }^{\mathbf{1}}$ Carolyn P. Sawicki, ${ }^{\mathbf{1}}$ Jacquie R. Baker, ${ }^{\mathbf{1}}$ and J. Kevin Shoemaker ${ }^{\mathbf{1 , 2}}$ \\ ${ }^{1}$ School of Kinesiology, Western University, London, Ontario, Canada; ${ }^{2}$ Department of Physiology and Pharmacology, \\ Western University, London, Ontario, Canada
}

Submitted 9 September 2013; accepted in final form 6 December 2013

\begin{abstract}
Lalande S, Sawicki CP, Baker JR, Shoemaker JK. Effect of age on the hemodynamic and sympathetic responses at the onset of isometric handgrip exercise. J Appl Physiol 116: 222-227, 2014. First published December 12, 2013; doi:10.1152/japplphysiol.01022.2013.—Cardiac and peripheral vasomotor factors contribute to the rapid pressor response at the onset of isometric handgrip exercise. We tested the hypothesis that age enhances the sympathetic and vasoconstrictor response at the onset of isometric handgrip exercise so that the pressor response is maintained, despite a diminished cardiac function. Twelve young and twelve older $(24 \pm 3$ and $63 \pm 8 \mathrm{yr})$ individuals performed 20 -s isometric handgrip exercise at 30,40 , or $50 \%$ of maximal voluntary contraction force. Muscle sympathetic nerve activity (MSNA) was measured using microneurography. Mean arterial pressure (MAP) and cardiac output $(\dot{\mathrm{Q}})$ were assessed continuously by finger plethysmography and total peripheral resistance was calculated. MAP increased with the onset of handgrip; this increase was associated with handgrip intensity and was similar in both groups. Heart rate and $\dot{Q}$ increased with increasing handgrip intensity in both groups, but increases were greater in young vs. older individuals (age $\times$ handgrip intensity interaction, $P<0.05)$. MSNA burst frequency increased $(P<0.01)$, while MSNA burst incidence tended to increase $(P=0.06)$ with increasing handgrip intensity in both groups. The change in MSNA between baseline and handgrip, for both frequency and incidence, increased with increasing handgrip intensity for both groups. There was no effect of handgrip intensity or age on total peripheral resistance. The smaller heart rate and Q response during the first $20 \mathrm{~s}$ of handgrip exercise in older individuals was not accompanied by a greater sympathetic activation or vasoconstrictor response. However, increases in MAP were similar between groups, indicating that the pressor response at the onset of handgrip exercise is preserved with aging.
\end{abstract}

aging; blood pressure regulation; exercise onset

ISOMETRIC HANDGRIP EXERCISE increases mean arterial pressure (MAP) $(5,11)$ through a coordinated series of reflexes emanating from central brain sites associated with volitional effort and from muscle sensory afferents $(14,28)$. This exerciseinduced pressor response begins at the very onset of the handgrip contraction, and the mechanisms of this rapid rise in MAP have been primarily related to changes in cardiac output $(22,24)$. A neurovascular component to the rise in MAP has also been proposed (19) on the basis of a large sympathetic response to fatiguing handgrip, although the association to the vascular response has been questioned (21). Nonetheless, a large sympathetic response occurs within 20-30 s of moderateintensity isometric handgrip exercise (7). The rapid cardiac response observed with handgrip exercise has been primarily related to parasympathetic withdrawal $(8,27)$, whereas the

Address for reprint requests and other correspondence: J. K. Shoemaker, Faculty of Health Sciences, Western Univ., Rm. 3110 Thames Hall, London, ON, Canada N6A 5B9 (e-mail: kshoemak@uwo.ca). somewhat delayed sympathetic response has been associated with a metabolites-mediated reflex from the fatiguing skeletal muscle $(13,27)$. Thus, unlike the multimodal contributions to blood pressure control during sustained handgrip exercise, the onset of isometric handgrip exercise (i.e., the first $20 \mathrm{~s}$ ) results in a rapid pressor response that mirrors the tachycardia with minimal change in sympathetic outflow (27).

The pressor response to fatiguing handgrip exercise is unaffected by age $(9,10,15,16,26)$. However, advancing age does attenuate the heart rate response to handgrip exercise $(9$, $10,15,16,26)$, possibly due to the decline in parasympathetic withdrawal observed in this population (25). The attenuated heart rate response and the resulting blunted cardiac output in older individuals (10) could, therefore, reduce the cardiac contribution to the increase in MAP at the onset of handgrip exercise. Thus we tested the hypothesis that age enhances the sympathetic and vasoconstrictor response at the onset of isometric handgrip exercise so that the pressor response is sustained in the presence of diminished cardiac function. To address this hypothesis, we measured the hemodynamic and muscle sympathetic nerve activity (MSNA) responses to 20-s bouts of isometric handgrip exercise performed at 30, 40, and $50 \%$ of maximal voluntary contraction (MVC) in young and older individuals.

\section{METHODS}

Twelve young and twelve older individuals with no history of cardiac, metabolic, neurological, inflammatory, or psychological disease and who were nonsmokers participated in the study. The study protocol was approved by the Office of Research Ethics at Western University, and all participants provided written, informed consent for participation in the study. Participants refrained from caffeine and heavy exercise for $12 \mathrm{~h}$ before arriving at the laboratory. Height and weight were recorded before participants assumed the supine position on a bed. Baseline systolic (SBP) and diastolic blood pressures (DBP) were measured using manual sphygmomanometry, and an average of three measures was calculated. Participants were first asked to perform two maximal voluntary handgrip contractions (MVC), and the largest of these contractions was used to determine the relative intensity of the handgrip exercise. Participants performed four 20-s handgrips at $30 \%$ of MVC, with each handgrip separated by $1 \mathrm{~min}$ of rest. The same handgrip protocol was also performed at 40 and $50 \%$ of MVC. The 30 and $40 \%$ MVC intensities were performed in a random order, while $50 \% \mathrm{MVC}$ intensity was always performed last. Participants reported their rate of perceived exertion at the end of each group of four handgrips using the Borg scale (2). Participants were instructed to breathe spontaneously during handgrip exercise, and all handgrips were performed with the left arm, which was the nondominant arm in all but two older participants.

Heart rate was measured from a three-lead electrocardiogram (Pilot, Colin Medical Instruments, San Antonio, TX). Mean arterial pressure (MAP), SBP, and DBP were measured continuously by 
finger plethysmography (Finometer, Finapres Medical Systems, Amsterdam, the Netherlands) on the middle finger of the nonexercising hand. Pulse pressure (PP), a surrogate marker for arterial stiffness, was calculated as the difference between systolic and diastolic pressures. Stroke volume and cardiac output were assessed by finger plethysmography using the Modelflow method (1). Total peripheral resistance was calculated as the quotient of MAP and cardiac output. Baseline beat-by-beat hemodynamic measures were averaged over the 30 -s preceding each handgrip. An average of the four baseline measures preceding each of the four handgrips performed at $30 \%$ of MVC was calculated. Similarly, averages of the four baseline measures at 40 and $50 \%$ of MVC were calculated. Since there was no difference between baseline values at 30, 40, and 50\% of MVC, an average baseline value for all handgrip intensities was calculated and reported. Hemodynamic measures were collected in the last $10 \mathrm{~s}$ of each handgrip, and all four handgrip measures performed at a given handgrip exercise intensity were reported as an average value for each individual.

MSNA was measured from the right peroneal nerve using microneurography (6). Briefly, a tungsten microelectrode was inserted percutaneously into the fibular nerve posterior to the fibular head. A suitable sympathetic nerve site was determined by a characteristic pulse-synchronous burst pattern. The MSNA neurogram was amplified 1,000 times through a preamplifier and 100 times by a variablegain, isolated amplifier. The amplified raw MSNA signal was bandpass filtered at a bandwidth of $700-2,000 \mathrm{~Hz}$, sampled at $10,000 \mathrm{~Hz}$, and stored offline for further analysis (Powerlab, ADInstruments, Colorado Springs, CO). Integrated bursts of MSNA were identified as exhibiting pulse synchrony, having a signal-to-noise ratio of at least $2: 1$ with respect to the previous period of neural silence between bursts and having characteristic rising and falling slopes. Burst occurrence was confirmed by visually inspecting the corresponding raw neurogram. Baseline burst frequency (bursts/min) and incidence (bursts/100 heartbeats) were obtained in the 30-s preceding each handgrip. Total MSNA was calculated as the product of mean burst area per minute and burst frequency. No difference was observed in baseline burst frequency and incidence over the course of the study (data not shown). Therefore, the four baseline measures for each handgrip intensities were average and reported as one baseline value. Exercise MSNA burst frequency and incidence were obtained over the $20 \mathrm{~s}$ of handgrip exercise, and the values from the four bouts of each handgrip intensity were averaged.

Statistics. Comparisons of baseline characteristics between young and older groups were conducted using a Student's unpaired $t$-test. A mixed one-way analysis of variance was used to test the effect of handgrip intensity and age. Post hoc analyses were performed using Bonferroni correction. Pearson's correlation coefficient was used for the analysis of associations between variables. Multiple linear regressions were performed to determine the independent predictors of the MAP response to isometric handgrip exercise in both young and older individuals. Results are expressed as means \pm SD. $P$ values $<0.05$ were considered statistically significant.

\section{RESULTS}

Young and older individuals had similar height, weight, and resting heart rate, while older individuals had greater SBP and DBP than young individuals (Table 1). Both groups had similar MVCs $[0.22 \pm 0.02$ vs. $0.22 \pm 0.04 \mathrm{~V}$ in young vs. older individuals, respectively; $P=$ nonsignificant (NS)]. Isometric handgrip exercise was performed at $28 \pm 1$ and $30 \pm 1 \%$ MVC, $37 \pm 1$ and $39 \pm 1 \% \mathrm{MVC}$, and $47 \pm 2$ and $49 \pm 2 \%$ MVC in young and older individuals, respectively $(P=\mathrm{NS})$. In accordance, the reported rates of perceived exhaustion were $11 \pm 2$ and $11 \pm 2,12 \pm 2$ and $12 \pm 2$, and $14 \pm 3$ and $14 \pm$
Table 1. Participants' characteristics

\begin{tabular}{lcc}
\hline \hline & Young & Older \\
\hline No. of subjects & $12(5$ men $)$ & $12(7$ men $)$ \\
Age, yr & $24 \pm 3$ & $63 \pm 8^{*}$ \\
Height, cm & $169 \pm 8$ & $168 \pm 10$ \\
Weight, kg & $67 \pm 16$ & $69 \pm 13$ \\
Heart rate, beats/min & $63 \pm 7$ & $61 \pm 7$ \\
SBP, mmHg & $111 \pm 10$ & $123 \pm 19^{*}$ \\
DBP, mmHg & $67 \pm 7$ & $73 \pm 8^{*}$ \\
\hline
\end{tabular}

Values are means $\pm \mathrm{SD}$. SBP, systolic blood pressure; DBP, disastolic blood pressure. *Different from young subjects, $P<0.05$.

2 across the three handgrip intensities in young vs. older individuals, respectively $(P=\mathrm{NS})$.

Baseline MAP was higher in older vs. young individuals, but increased similarly in both groups in a manner that was graded with handgrip intensity (Table 2). Similarly, SBP and DBP were higher in older vs. young individuals and increased with handgrip intensity in both groups (Table 2). There was an age $\times$ handgrip intensity interaction for PP, where PP increased with increasing handgrip intensity in older individuals, but not in young individuals (Table 2 ). There was an age $\times$ handgrip intensity interaction for heart rate (Table 2), where heart rate increased with increasing handgrip intensity in both groups, but the increase was greater in young vs. older individuals (Fig. 1). Also, an age $\times$ handgrip intensity interaction was observed for cardiac output (Table 2), where cardiac output increased with handgrip intensity in both groups but more in young vs. older individuals (Fig. 1). The increase in cardiac output with handgrip exercise was due to the heart rate response, as stroke volume did not increase in either group (Table 2). Total peripheral resistance also did not change with handgrip exercise in either group (Table 2).

As expected, baseline MSNA burst frequency and burst incidence were greater in older vs. young individuals (main effect of group for burst frequency: $P=0.002$, and burst incidence: $P=0.001$, Table 2). During isometric handgrip exercise, burst frequency increased with increasing handgrip intensity (main effect of handgrip: $P=0.004$ ), while burst incidence only tended to increase (main effect of handgrip: $P=0.06$, Table 2). A main effect of handgrip intensity on the changes in burst frequency was observed as well $(P=0.002$, Fig. 2). An age $\times$ handgrip intensity interaction $(P=0.02)$ was observed for the handgrip-induced changes in burst incidence (Fig. 2). Notably, the average changes in burst incidence decreased below baseline at the lower handgrip intensity in older individuals. The effects of handgrip intensity, as well as the age $\times$ handgrip intensity interaction, were also observed when changes in burst frequency and burst incidence were expressed in relative values. Similar to baseline MSNA burst frequency and burst incidence, total MSNA was greater in older vs. young individuals at baseline and for all handgrip intensities (main effect of group: $P=0.03$, Table 2). Moreover, total MSNA also increased with increasing handgrip intensity (main effect of handgrip: $P=0.019$ ). However, relative changes in total MSNA with increasing handgrip intensity were not different between groups (young: $-6 \pm 45$, $15 \pm 79$, and $166 \pm 219 \%$; older: $15 \pm 33,29 \pm 41$, and $75 \pm$ $80 \%$ at 30,40 , and $50 \%$ of MVC, respectively).

Even though the average total peripheral resistance did not change with handgrip exercise in either group, changes in total 
Table 2. Hemodynamic and sympathetic response at the onset of isometric handgrip exercise performed at 30, 40, and 50\% of MVC in young and older individuals

\begin{tabular}{|c|c|c|c|c|c|c|c|}
\hline & Baseline & $30 \%$ & $40 \%$ & $50 \%$ & Handgrip Intensity & Group & Interaction \\
\hline \multicolumn{8}{|c|}{ MAP, mmHg } \\
\hline Young & $82 \pm 7$ & $81 \pm 7$ & $84 \pm 7$ & $86 \pm 7 * \dagger$ & \multirow{2}{*}{$<0.001$} & \multirow[t]{2}{*}{0.022} & \multirow[t]{2}{*}{0.089} \\
\hline Older & $90 \pm 11$ & $93 \pm 12$ & $94 \pm 13^{*}$ & $96 \pm 14^{* \dagger}$ & & & \\
\hline \multicolumn{8}{|c|}{$\mathrm{SBP}, \mathrm{mmHg}$} \\
\hline Young & $111 \pm 10$ & $110 \pm 10$ & $113 \pm 10$ & $115 \pm 11^{* \dagger}$ & \multirow[t]{2}{*}{$<0.001$} & \multirow[t]{2}{*}{0.035} & \multirow[t]{2}{*}{0.054} \\
\hline Older & $123 \pm 19$ & $127 \pm 22 *$ & $129 \pm 22 *$ & $131 \pm 24 * \dagger$ & & & \\
\hline \multicolumn{8}{|c|}{ DBP, $\mathrm{mmHg}$} \\
\hline Young & $67 \pm 7$ & $67 \pm 7$ & $69 \pm 9^{* \dagger}$ & $72 \pm 5^{* \dagger}$ & \multirow[t]{2}{*}{$<0.001$} & \multirow[t]{2}{*}{0.032} & \multirow[t]{2}{*}{0.333} \\
\hline Older & $73 \pm 8$ & $75 \pm 9$ & $77 \pm 10^{*}$ & $78 \pm 10^{* \dagger}$ & & & \\
\hline \multicolumn{8}{|l|}{$\mathrm{PP}, \mathrm{mmHg}$} \\
\hline Young & $44 \pm 6$ & $44 \pm 7$ & $44 \pm 7$ & $43 \pm 8$ & \multirow[t]{2}{*}{0.051} & \multirow[t]{2}{*}{0.102} & \multirow[t]{2}{*}{$<0.001$} \\
\hline Older & $50 \pm 15$ & $51 \pm 16$ & $52 \pm 17$ & $53 \pm 17$ & & & \\
\hline \multicolumn{8}{|l|}{$\dot{\mathrm{Q}}, 1 / \mathrm{min}$} \\
\hline Young & $5.9 \pm 1.5$ & $6.1 \pm 1.7$ & $6.4 \pm 1.7 * \dagger$ & $6.8 \pm 1.8^{* \dagger \neq}$ & \multirow[t]{2}{*}{$<0.001$} & \multirow[t]{2}{*}{0.283} & \multirow{2}{*}{0.033} \\
\hline Older & $5.4 \pm 1.1$ & $5.6 \pm 1.2$ & $5.8 \pm 1.3^{*}$ & $5.8 \pm 1.3^{*}$ & & & \\
\hline \multicolumn{8}{|c|}{ HR, beats/min } \\
\hline Young & $63 \pm 7$ & $65 \pm 6$ & $70 \pm 8^{* \dagger}$ & $77 \pm 11 * \dagger \neq$ & \multirow[t]{2}{*}{$<0.001$} & \multirow[t]{2}{*}{0.122} & \multirow[t]{2}{*}{0.023} \\
\hline Older & $61 \pm 7$ & $63 \pm 8$ & $64 \pm 8$ & $67 \pm 11 * \dagger$ & & & \\
\hline \multicolumn{8}{|l|}{$\mathrm{SV}, \mathrm{ml}$} \\
\hline Young & $94 \pm 25$ & $90 \pm 20$ & $90 \pm 21$ & $88 \pm 22$ & \multirow[t]{2}{*}{0.05} & \multirow[t]{2}{*}{0.739} & \multirow[t]{2}{*}{0.431} \\
\hline Older & $91 \pm 22$ & $92 \pm 25$ & $92 \pm 24$ & $88 \pm 24$ & & & \\
\hline TPR, mmF & & & & & & & \\
\hline Young & $14.8 \pm 3.6$ & $14.2 \pm 3.5$ & $13.9 \pm 3.5$ & $13.4 \pm 3.3$ & 0.133 & 0.111 & 0.109 \\
\hline Older & $17.8 \pm 6.5$ & $17.8 \pm 6.9$ & $17.7 \pm 7.1$ & $17.9 \pm 7.5$ & & & \\
\hline Burst frequ & & & & & & & \\
\hline Young & $11.5 \pm 5.0$ & $13.3 \pm 7.0$ & $13.6 \pm 7.3$ & $17.1 \pm 11.6^{*}$ & 0.004 & 0.002 & 0.632 \\
\hline Older & $25.3 \pm 9.6$ & $27.3 \pm 9.6$ & $28.7 \pm 14.0$ & $32.1 \pm 10.2^{*}$ & & & \\
\hline Burst incid & & & & & & & \\
\hline Young & $18.4 \pm 8.0$ & $20.9 \pm 11.5$ & $19.7 \pm 10.4$ & $23.7 \pm 15.0$ & 0.064 & 0.001 & 0.272 \\
\hline Older & $42.2 \pm 15.5$ & $39.6 \pm 16.8$ & $45.2 \pm 21.1$ & $49.4 \pm 16.1$ & & & \\
\hline Total MSN & & & & & & & \\
\hline Young & $10.9 \pm 6.9$ & $10.4 \pm 8.9$ & $13.2 \pm 10.7$ & $28.3 \pm 26.5$ & 0.019 & 0.029 & 0.333 \\
\hline Older & $48.1 \pm 48.4$ & $60.9 \pm 79.4$ & $73.3 \pm 99.8$ & $71.2 \pm 50.7$ & & & \\
\hline
\end{tabular}

Values are means \pm SD. MVC, maximal voluntary contraction; MAP, mean arterial pressure; PP, pulse pressure; $\dot{Q}$, cardiac output; HR, heart rate; SV, stroke volume; TPR, total peripheral resistance; MSNA, muscle sympathetic nerve activity. Different from *baseline, $\dagger 30 \%$ MVC, and $\ddagger 40 \%$ MVC: all $P<0.05$.

peripheral resistance were correlated with changes in burst frequency in older individuals $(r=0.33, P=0.05)$, but not in young individuals $(r=0.02, P=0.93)$ (Fig. 3). Multiple linear regressions revealed that total peripheral resistance $(P<0.01)$ and cardiac output $(P=0.01)$ were independent predictors of the MAP response to isometric handgrip exercise in young individuals, while total peripheral resistance $(P<0.01)$ and PP $(P<0.01)$ were independent predictors of the changes in MAP during isometric handgrip exercise in older individuals.

\section{DISCUSSION}

The present results indicate that the rapid increase in MAP observed at the onset of isometric handgrip exercise is preserved in older individuals, despite the diminished cardiac response observed in this group. Specifically, older individuals showed a smaller heart rate and cardiac output response to handgrip exercise compared with young individuals. Contrary to the hypothesis under investigation, a greater sympathetic or vasoconstrictor response was not observed in the older group. Thus, based on the greater PP response, we speculate that an increased arterial stiffness in older individuals enables a smaller change in cardiac output to induce greater changes in MAP in this population.

Whether the rise in blood pressure during handgrip exercise is driven primarily by elevations in cardiac output or by sympathetically induced increases in total peripheral resistance has been a common focus in reflex cardiovascular research. Previous explorations of this question have relied on more delayed or steady-state conditions that occur after 1-2 min of handgrip exercise. Using this latter approach, it was reported that static handgrip exercise results in elevated cardiac output with little change in systemic vascular resistance $(4,11,22)$. Indeed, during ischemic handgrip exercise, cardiac output accounted for increases in MAP due to elevations in heart rate and stroke volume, despite large increases in MSNA (22). However, the contributions of stroke volume may be delayed beyond the first $20 \mathrm{~s}$ of handgrip exercise $(22,24)$, such that the initial rapid increase in MAP and cardiac output are driven primarily by changes in heart rate.

This study focused on the first $20 \mathrm{~s}$ of isometric handgrip exercise to establish the neural and cardiac contributions to blood pressure at the onset of exercise. In this time period, the rise in heart rate accounted for the increase in cardiac output with little change in stroke volume. In accordance with previous studies $(9,10,15,16,26)$, we observed an attenuated heart rate response to isometric handgrip exercise in older individuals. The attenuated heart rate response in older individuals at the onset of handgrip exercise is consistent with a decline in parasympathetic withdrawal with aging due to either a reduced baseline level of cardiac vagal outflow (25) and/or to an age-related decline in cardiac $\mathrm{M}_{2}$ muscarinic receptor density and function (3). Regardless, the lack of change in average 

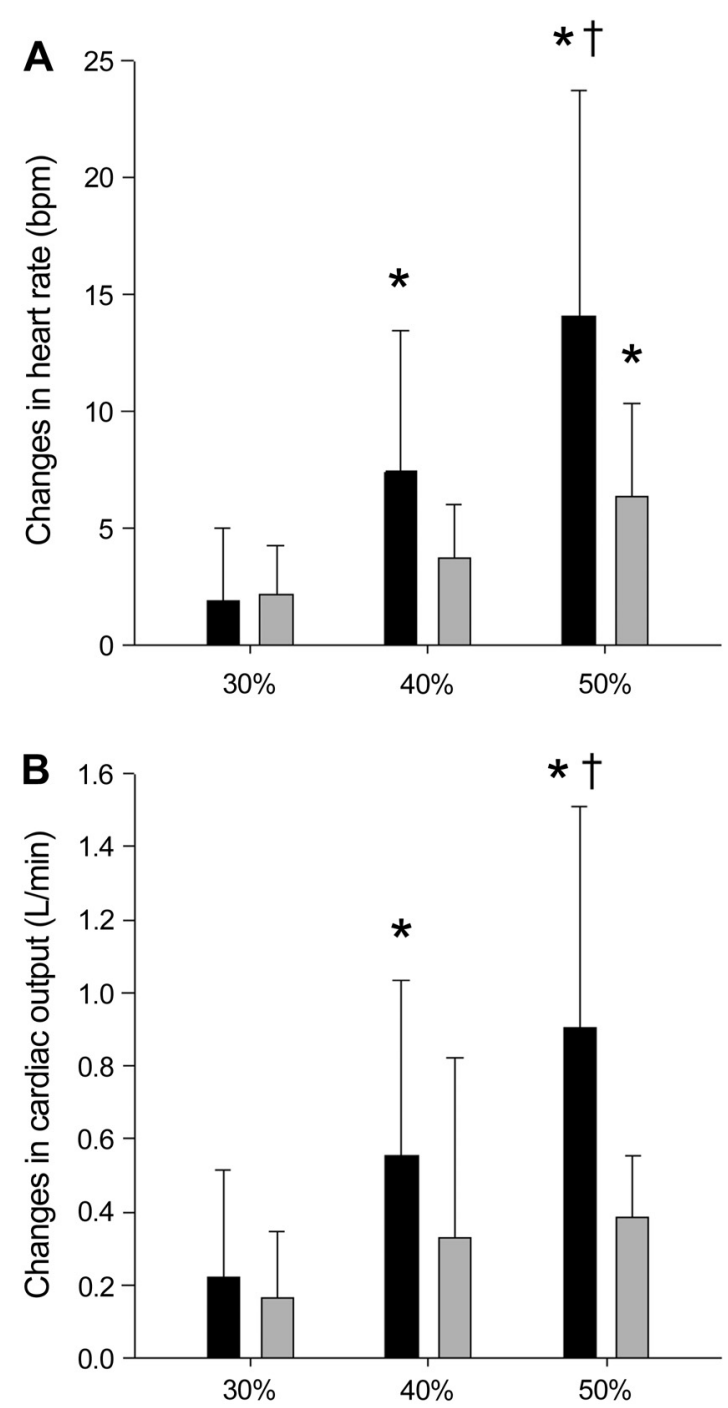

Fig. 1. Changes in heart rate $(A)$ and changes in cardiac output $(B)$ from baseline (BL) to 30,40 , and $50 \%$ of maximal voluntary contraction (MVC) in young (solid bars) and older individuals (shaded bars). There was a main effect for handgrip intensity $(P<0.01)$ and an age $\times$ handgrip intensity interaction $(P<0.05)$. *Different from $30 \%$ of MVC. $†$ Different from $40 \%$ of MVC.

total peripheral resistance in both groups suggests that cardiac output plays a greater role in increasing MAP at the onset of handgrip exercise than does vasoconstriction. However, this does not explain the similar increase in MAP, despite a lower cardiac output response in older individuals. Rather, an increased arterial stiffness with aging may contribute to the similar MAP response in both groups. This conjecture is supported by an observed increase in PP during $20 \mathrm{~s}$ of isometric handgrip exercise in the older individuals of the present study. Thus, while changes in cardiac output act as primary determinants of the pressor response in young individuals, an increased arterial stiffness contributes to the increase MAP during isometric handgrip exercise in older individuals.

Changes in MSNA burst frequency and burst incidence increased similarly with increasing handgrip intensity in both groups. A lack of difference in changes in MSNA between young and older individuals has previously been reported during sustained handgrip exercise until exhaustion, defined as the inability to maintain handgrip intensity and achievement of peak level of perceived exertion (16). The increase in burst frequency and burst incidence in the present study did not trigger any significant changes in average total peripheral resistance in both groups (Table 2). Despite the observed lack of change in average total peripheral resistance, variability exists in the total peripheral resistance response to increases in MSNA across individuals. Indeed, changes in total peripheral resistance were correlated, albeit weakly, to changes in burst frequency in older individuals, but not in young individuals. The lack of association between total peripheral resistance and MSNA in young individuals further supports the observed dissociation between MSNA and limb vascular resistance previously reported in this population $(18,21)$.

Interestingly, we observed increases in MSNA burst frequency within the first $20 \mathrm{~s}$ of isometric handgrip exercise. Typically, the increase in MSNA during isometric exercise is predominantly attributed to the metaboreflex from active muscles and is usually not observed during the first minute of
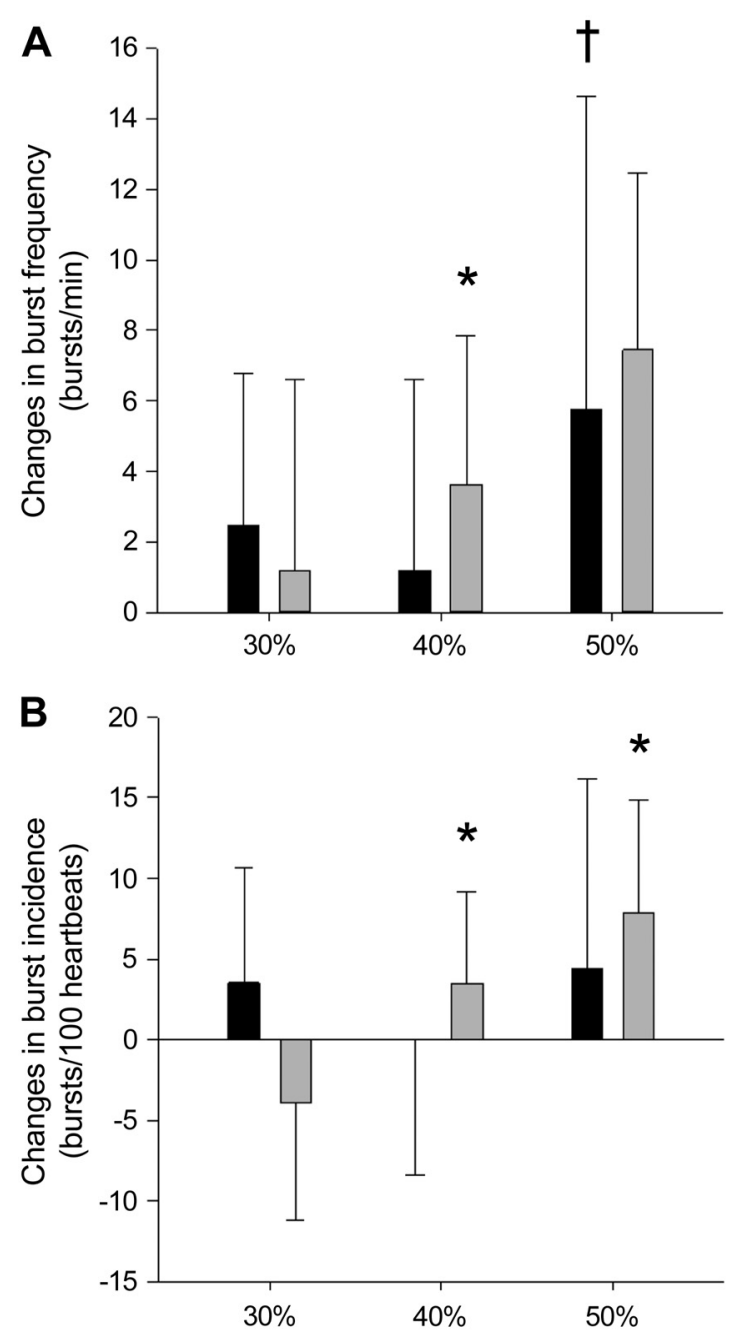

Fig. 2. Changes in burst frequency $(A)$ and changes in burst incidence $(B)$ from BL to 30,40 , and $50 \%$ of MVC in young (solid bars) and older individuals (shaded bars). There was a main effect for handgrip intensity $(P<0.05)$, and an age $\times$ handgrip intensity interaction $(P<0.05)$. *Different from $30 \%$ of MVC. $\dagger$ Different from $40 \%$ of MVC. 

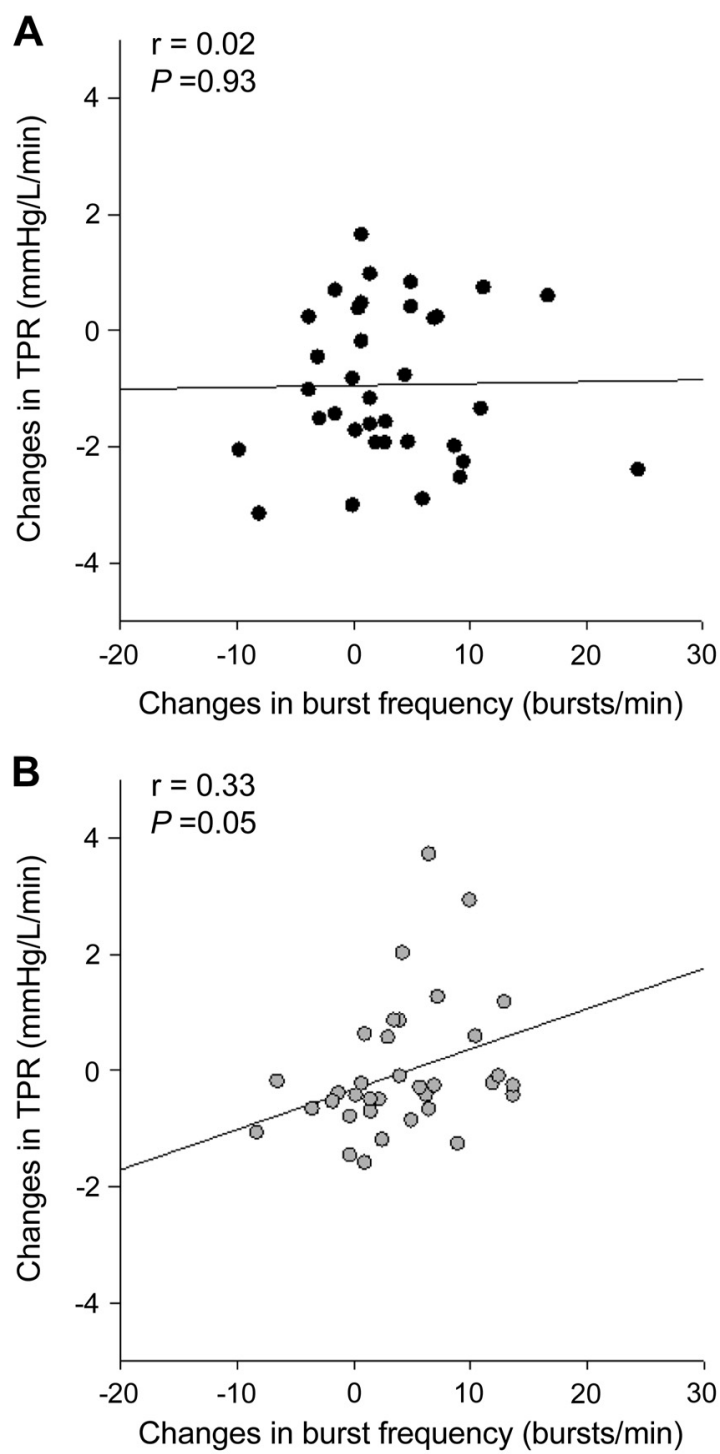

Fig. 3. Correlations between changes in burst frequency and changes in total peripheral resistance (TPR) in young $(A)$ and older $(B)$ individuals at all handgrip intensities.

isometric exercise performed at $30 \% \operatorname{MVC}(13,27)$. This latency period before the increase in MSNA appears to be intensity dependent, with a greater exercise intensity corresponding to a shorter latency period (17). When participants were asked to perform handgrip with maximal effort for $2 \mathrm{~min}$, which was equivalent to $58 \% \mathrm{MVC}$ after $10 \mathrm{~s}$ of exercise, MSNA increased $20 \mathrm{~s}$ after the onset of handgrip exercise (7). It was concluded that differences in timing of metaboreflex activation depended on the speed of accumulation of metabolites in the contracting muscle $(13,20)$. Moreover, sympathetic blockade depressed the heart rate response to isometric handgrip exercise after $10 \mathrm{~s}$ of contraction at 50 and $75 \% \mathrm{MVC}$, supporting sympathetic contribution to the heart rate response at the beginning of heavier exercise (12). Therefore, effort levels above $50 \%$ of MVC seem to activate sympathetic efferent outflow within a short period of time. These previous findings support a sympathetic activation within the first $20 \mathrm{~s}$ of handgrip exercise, with observed increases in MSNA with increasing handgrip intensity. It is also of interest to note that greater increases in MSNA have been reported during isometric handgrip performed by the nondominant vs. dominant forearm (23), and that handgrip exercise was performed with the nondominant forearm for the majority of our participants. In this study, exercise bouts of $20 \mathrm{~s}$ were used in the attempt to avoid metaboreflex activation. We used a resting period of 1 min between each handgrip, which raised a possible concern that there was metabolite built up with repeated handgrip exercise. To minimize this possibility, the order of 30 and $40 \%$ of MVC handgrip exercise was randomized, and handgrip exercise at 50\% of MVC was always performed last. Moreover, there were no changes in baseline MSNA at each handgrip intensity, suggesting that neither accumulation of metabolites or increased sensitivity of the metaboreceptors occurred between each handgrip.

The present study focused on the hemodynamic and sympathetic responses to 20-s bouts of isometric handgrip exercise, representing a relatively short time period to measure MSNA. Nonetheless, the MSNA burst frequency across the various handgrip intensities was quite homogeneous across individuals, both in the young and older group (Fig. 4). Therefore, we
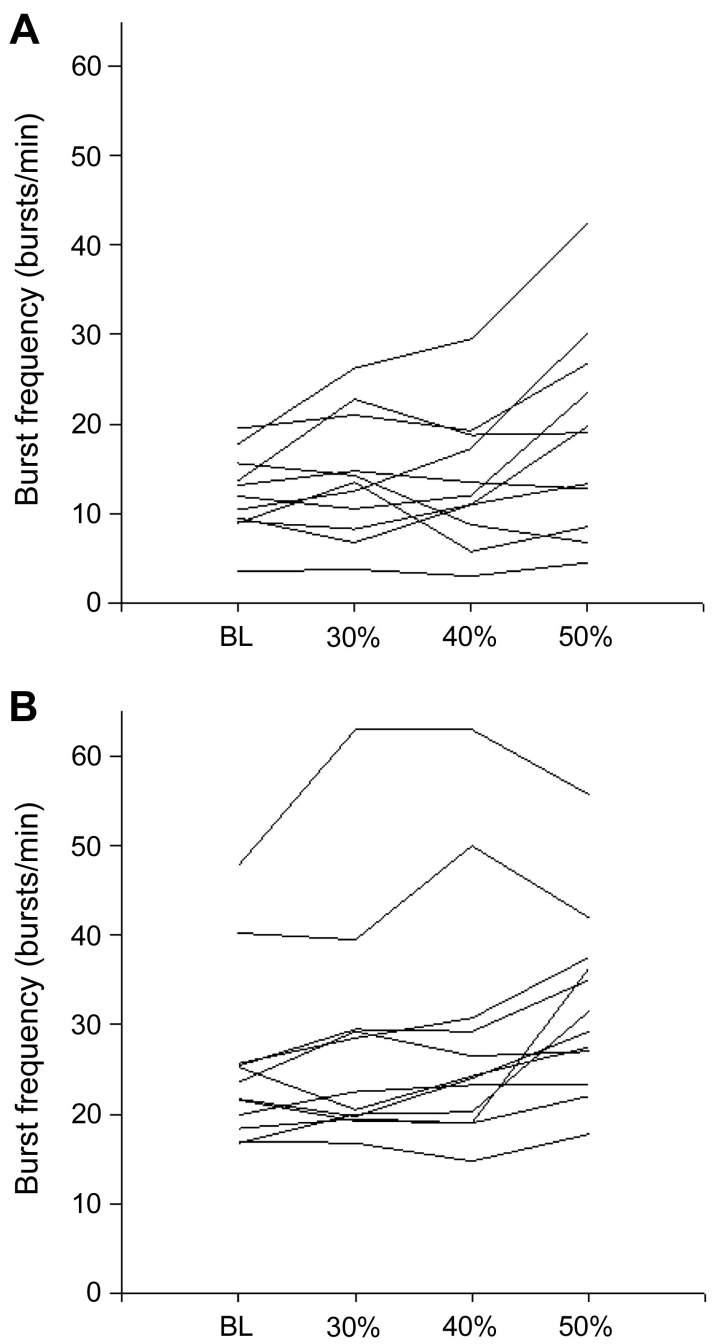

Fig. 4. Muscle sympathetic nerve activity burst frequency at BL and across all handgrip intensities in young $(A)$ and older $(B)$ individuals. 
do not believe that excessive interindividual variability or between-contraction variations precluded detection of any real differences. In summary, older and young individuals had similar increases in MAP at the onset of isometric handgrip exercise, despite an attenuated heart rate and cardiac output response to handgrip exercise in older individuals. Despite an increase in MSNA burst frequency, there was no change in average total peripheral resistance in either young or older group. It appears that a stiffer vascular system in older individuals facilitates a conserved pressor response to handgrip exercise, despite modulation of cardiac function.

\section{ACKNOWLEDGMENTS}

We thank Carly Barron, Arlene Fleischhauer, and Maria Frances for excellent technical support.

\section{GRANTS}

This study was supported by the Natural Sciences and Engineering Research Council of Canada and the Canadian Institutes of Health Research Team Grant "Physical Activity, Mobility and Neural Health" (Grant 217532) to J. K. Shoemaker. J. K. Shoemaker is a Canada Research Chair.

\section{DISCLOSURES}

No conflicts of interest, financial or otherwise, are declared by the author(s).

\section{AUTHOR CONTRIBUTIONS}

Author contributions: S.L. and J.R.B. performed experiments; S.L. and C.P.S. analyzed data; S.L. and J.K.S. interpreted results of experiments; S.L. prepared figures; S.L. drafted manuscript; S.L., C.P.S., J.R.B., and J.K.S. edited and revised manuscript; S.L., C.P.S., J.R.B., and J.K.S. approved final version of manuscript; J.K.S. conception and design of research.

\section{REFERENCES}

1. Bogert LW, van Lieshout JJ. Non-invasive pulsatile arterial pressure and stroke volume changes from the human finger. Exp Physiol 90: 437-446, 2005.

2. Borg G. Perceived exertion as an indicator of somatic stress. Scand $J$ Rehabil Med 2: 92-98, 1970.

3. Brodde OE, Konschak U, Becker K, Ruter F, Poller U, Jakubetz J, Radke J, Zerkowski HR. Cardiac muscarinic receptors decrease with age. In vitro and in vivo studies. J Clin Invest 101: 471-478, 1998.

4. Crisafulli A, Scott AC, Wensel R, Davos CH, Francis DP, Pagliaro P, Coats AJ, Concu A, Piepoli MF. Muscle metaboreflex-induced increases in stroke volume. Med Sci Sports Exerc 35: 221-228; discussion 229, 2003.

5. Delius W, Hagbarth KE, Hongell A, Wallin BG. Manoeuvres affecting sympathetic outflow in human muscle nerves. Acta Physiol Scand 84: 82-94, 1972.

6. Hagbarth KE, Vallbo AB. Pulse and respiratory grouping of sympathetic impulses in human muscle-nerves. Acta Physiol Scand 74: 96-108, 1968.

7. Hashimoto I, Miyamura M, Saito M. Initiation of increase in muscle sympathetic nerve activity delay during maximal voluntary contraction. Acta Physiol Scand 164: 293-297, 1998.

8. Hollander AP, Bouman LN. Cardiac acceleration in man elicited by a muscle-heart reflex. J Appl Physiol 38: 272-278, 1975.
9. Houssiere A, Najem B, Pathak A, Xhaet O, Naeije R, Van De Borne P. Chemoreflex and metaboreflex responses to static hypoxic exercise in aging humans. Med Sci Sports Exerc 38: 305-312, 2006.

10. Krzeminski K, Cybulski G, Ziemba A, Nazar K. Cardiovascular and hormonal responses to static handgrip in young and older healthy men. Eur J Appl Physiol 112: 1315-1325, 2012.

11. Lind AR, Taylor SH, Humphreys PW, Kennelly BM, Donald KW. The circulatiory effects of sustained voluntary muscle contraction. Clin Sci (Lond) 27: 229-244, 1964.

12. Maciel BC, Gallo Junior L, Marin Neto JA, Martins LE. Autonomic nervous control of the heart rate during isometric exercise in normal man. Pflügers Arch 408: 173-177, 1987.

13. Mark AL, Victor RG, Nerhed C, Wallin BG. Microneurographic studies of the mechanisms of sympathetic nerve responses to static exercise in humans. Circ Res 57: 461-469, 1985.

14. Mitchell JH, Kaufman MP, Iwamoto GA. The exercise pressor reflex: its cardiovascular effects, afferent mechanisms, and central pathways. Aпnи Rev Physiol 45: 229-242, 1983.

15. Muller MD, Gao Z, Mast JL, Blaha CA, Drew RC, Leuenberger UA, Sinoway LI. Aging attenuates the coronary blood flow response to cold air breathing and isometric handgrip in healthy humans. Am J Physiol Heart Circ Physiol 302: H1737-H1746, 2012.

16. Ng AV, Callister R, Johnson DG, Seals DR. Sympathetic neural reactivity to stress does not increase with age in healthy humans. Am J Physiol Heart Circ Physiol 267: H344-H353, 1994.

17. Ray CA, Allyn LM. Sympathetic adjustments to exercise: insights from microneurographic recordings. In: Cardiovascular Reflex Control in Health and Disease, edited by Hainsworth R and Allyn LM. Philadelphia, PA: Saunders, 1993.

18. Saito M, Mano T, Iwase $\mathbf{S}$. Changes in muscle sympathetic nerve activity and calf blood flow during static handgrip exercise. Eur J Appl Physiol Occup Physiol 60: 277-281, 1990.

19. Seals DR. Sympathetic neural discharge and vascular resistance during exercise in humans. J Appl Physiol 66: 2472-2478, 1989.

20. Seals DR, Enoka RM. Sympathetic activation is associated with increases in EMG during fatiguing exercise. J Appl Physiol 66: 88-95, 1989.

21. Shoemaker JK, Herr MD, Sinoway LI. Dissociation of muscle sympathetic nerve activity and leg vascular resistance in humans. Am J Physiol Heart Circ Physiol 279: H1215-H1219, 2000.

22. Shoemaker JK, Mattar L, Kerbeci P, Trotter S, Arbeille P, Hughson RL. WISE 2005: stroke volume changes contribute to the pressor response during ischemic handgrip exercise in women. J Appl Physiol 103: $228-$ 233, 2007.

23. Sinoway LI, Rea RF, Mosher TJ, Smith MB, Mark AL. Hydrogen ion concentration is not the sole determinant of muscle metaboreceptor responses in humans. J Clin Invest 89: 1875-1884, 1992.

24. Stewart JM, Montgomery LD, Glover JL, Medow MS. Changes in regional blood volume and blood flow during static handgrip. Am J Physiol Heart Circ Physiol 292: H215-H223, 2007.

25. Stratton JR, Levy WC, Caldwell JH, Jacobson A, May J, Matsuoka D, Madden K. Effects of aging on cardiovascular responses to parasympathetic withdrawal. J Am Coll Cardiol 41: 2077-2083, 2003.

26. Taylor JA, Hand GA, Johnson DG, Seals DR. Sympathoadrenalcirculatory regulation during sustained isometric exercise in young and older men. Am J Physiol Regul Integr Comp Physiol 261: R1061-R1069, 1991.

27. Victor RG, Seals DR, Mark AL. Differential control of heart rate and sympathetic nerve activity during dynamic exercise. Insight from intraneural recordings in humans. J Clin Invest 79: 508-516, 1987.

28. Williamson JW, Fadel PJ, Mitchell JH. New insights into central cardiovascular control during exercise in humans: a central command update. Exp Physiol 91: 51-58, 2006. 